\title{
Creación de un jardín botánico y de árbol madre de arbustivas forrajeras nativas del estado de Sonora
}

\author{
Creation of a botanical garden and mother tree of forage shrub \\ native to the state of Sonora \\ Diana M. Mc Caughey-Espinoza1, Gloria Irma Ayala Astorga ${ }^{1}$, \\ Juvenal Velázquez-Caudillo², Jesús Anaya-Islas ${ }^{2}$ y Eduardo Canseco-Vilchis ${ }^{2}$
}

\begin{abstract}
RESUMEN
El estado Mexicano de Sonora cuenta con una gran diversidad florística, de gran valor forrajero que actualmente se encuentran en peligro de desaparecer o disminuir sus poblaciones, debido al mal manejo de los agostaderos y el cambio de uso de suelo (urbanización, agrícola, acuícola, industria minera y cementera, etc.), provocando serios problemas a los ecosistemas naturales. El objetivo de nuestro trabajo fue crear un jardín y de árbol madre de arbustivas forrajeras del estado de Sonora. Se acondicionó una área de 2 ha y se le instaló un sistema de riego por goteo. Las especies involucradas fueron 10 arbustivas y 10 árboles, con edad promedio de dos años. La sobrevivencia al trasplante, de las especies arbustivas fue de 70 al $90 \%$ y los árboles presentaron del 60 al $100 \%$. Las arbustivas presentaron diámetros de tallos de 6,20 a $20,76 \mathrm{~cm}^{2}$, diámetros de copas de 0,63 a 2,11 $\mathrm{m}^{2}$, alturas de 0,45 a 1,62 metros y el índice de crecimiento de los diámetros de tallos fue de 22,3 a $43,2 \%$, coberturas de copas fue 28,8 a $67,4 \%$ con alturas de 17,0 hasta 48,6\%. Los árboles tuvieron diámetros de tallos de 0,85 a $31,93 \mathrm{~cm}^{2}$, diámetros de copas de 0,62 a $2,82 \mathrm{~m}^{2}$, alturas de 0,47 a 1,97 metros y el índice de crecimiento para los diámetros de tallos fue desde 15,2 a 77.0\%, con coberturas de copas 12,9 a $61,5 \%$ y alturas 7,7 a 82,5\%. Con la aplicación de los riegos, algunas especies presentaron floración de 3 a 4 veces al año. Palabras claves: jardín botánico, plantas forrajeras, arbustivas.
\end{abstract}

\section{ABSTRACT}

The Mexican state of Sonora has a great diversity of floristic plant species with great fodder value that are currently in danger of disappearing or diminishing their populations, due to the bad handling of the rangelands, and the change of land use (urbanization, agriculture, aquaculture, mining industry and cement etc.), causing serious problems to the natural ecosystems. Our work was done to establish a botanical garden and mother tree of forage shrubs of the state of Sonora. An area of 2 hectares was conditioned and a drip irrigation system was installed. The involved species were 10 shrubs and 10 trees with an average age of 2 years. The transplant survival of the shrub species was 70 to $90 \%$ and the trees presented from 60 to $100 \%$. The bush trees reached 6.20 to $20.76 \mathrm{~cm}^{2}$ of stem diameter, a crown diameter from 0.63 to $2.11 \mathrm{~m}^{2}$ and a height from 0.45 to $1.62 \mathrm{~m}$. The growth rate of the stem diameter was from 22.3 to 43.2 , its crown from 28.8 to 67.4 and the heights from 17.0 to $48.6 \%$. On the other hand, the trees stem diameters reached from 0.85 to $31.93 \mathrm{~cm}^{2}$, the crown diameters from 0.62 to $2.82 \mathrm{~m}^{2}$, and heights of 0.47 to 1.97 meters. Their growth rate for the stem diameter was from 15.2 to $77.0 \%$, the crowns 12.9 to $61.5 \%$ and heights from 7.7 to $82.5 \%$. With the irrigation systems, some species presented flowering from 3 to 4 times during the year.

Key words: Botanic garden, forage plants, shrubs.

\section{Introducción}

En México, existen diferentes ecosistemas naturales con una gran variación en sus características fisiográficas, donde los sistemas de producción pecuaria se han tenido que adaptar a las condiciones propias de cada ecosistema. Destacando, por su importancia y productividad,

1 Departamento de Investigaciones Científicas y Tecnológicas de la Universidad de Sonora. Hermosillo Sonora, México.

2 Departamento de Agricultura y Ganadería. Universidad de Sonora. Hermosillo Sonora, México.

* Autor de correspondencia: diana.mccaughey@unison.mx

Fecha de Recepción: 25 Mayo, 2017.

Fecha de Aceptación: 4 Septiembre, 2017.

DOI: 
las zonas tropicales, templadas, áridas y semiáridas. Sonora cuenta con con 18.493 .400 hectáreas de superficie total, las que están distribuidas en 23 tipos de eliminar diferentes y donde existe 16.170.500 ha, que son de agostadero natural (Jaramillo, 1994). Los agostaderos dedicados a la producción animal, se encuentran seriamente deteriorados y su alto potencial productivo ha bajado considerablemente. Entre un 30 y $70 \%$ de las especies nativas y de alto valor forrajero, y que actualmente presentan una fuerte disminución en sus poblaciones, tal es el caso del palo fierro (Olneya tesota A. Gray), zámota (Coursetia glandulosa A. Gray), cósahui (Krameria parvifolia Benth), entre otras, las que han tenido un notorio consumo por los animales domésticos y silvestres, aunado a los desmontes masivos y el cambio de uso de suelo, no les ha permitido propagarse en forma natural, por lo que se encuentran en serios problemas que los podría llevar a desaparecer de sus propios ecosistemas.

El 83\% del área total del estado de Sonora está siendo utilizada en programas de explotación ganadera, en donde alrededor de cinco millones de hectáreas, entre las que destacan el matorral arbosufrutescente, arborescente y alto espinoso, han sido afectadas en diferentes años por los desmontes totales y parciales para establecer praderas de zacate buffel (Cenchrus ciliaris L.), muchas de estas han sido abandonadas por haberse establecido en sitios no aptos o por mal manejo. Ibarra et al. (2005), estiman que existe por lo menos 1 millón de has, que presentan dichos problemas lo que ha bajado considerablemente la producción de forrajera (150-300 kg/ha) al producir de 3 a 5 veces menos forraje. Las especies que fueron eliminadas, han sido sustituidas por especies invasoras con baja calidad nutricional, como el caso del chírahui (Acacia cochliocantha Willd), gobernadora (Larrea tridentata (Sesse y Moc Ex Dc) Coville) y rama blanca (Encelia farinosa Torr y A. Gray). Ante esta deplorable situación, es necesario establecer programas integrales de reforestación, que permita hacer una revegetación natural y artificial, utilizando especies arbustivas forrajeras nativas que presenten buenos índices de adaptación y alta capacidad de producción. Sin embargo, la revegetación natural está determinada por las oportunidades de las especies para diseminarse y del manejo que se les dé con el pastoreo del ganado, el que debe de estar encaminado a la conservación de especies vegetales.
La realidad antes mencionada, demuestra que difícilmente se puede esperar una recuperación a corto plazo de estas áreas, aun cuando los bancos de germoplasma de estas especies existen en forma artificial o natural. Las semillas de plantas de interés alimenticio en la ganadería de la región presentan una baja disponibilidad en los bancos de semillas artificiales. Algunas semillas se encuentran bajo la superficie del suelo esperando las condiciones propicias para germinar, como también ya sabemos que parte de la producción de semillas son consumidas por los animales domésticos y silvestres. Las semillas que existen, en nuestros ecosistemas presentan inhibidores para su germinación, por tanto, requieren tratamientos pregerminativos, lo que también impide que existan nuevos ejemplares en los ecosistemas en forma natural, y la baja disponibilidad de semillas en los bancos de germoplasma artificiales no son suficientes para llevar acabo siembras masivas en los agostaderos, y que los costos de rehabilitación son muy altos, por lo que deben realizar esfuerzos para buscar nuevas estrategias y mejorando así, las expectativas de estos ecosistemas. Por las razones anteriormente expuestas, uno de los objetivos en el desarrollo de este estudio fue lograr el establecimiento de un jardín botánico de árbol madre con especies de arbustivas forrajeras nativas de Sonora, con un sistema de riego presurizado, que permitió tener una fuente segura de semillas de las especies establecidas y de esa manera promover los programas de reforestación en las áreas más deterioradas del estado de Sonora.

\section{Materiales y Métodos}

Ubicación del área de estudio. El presente trabajo de investigación se inicio en el mes de agosto de 2001 a diciembre del 2002. Se llevó a cabo en el Campo Experimental del Departamento de Agricultura y Ganadería de la Universidad de Sonora, localizado en el $\mathrm{km} 21$ de la carretera Hermosillo a Bahía de Kino, el que se encuentra a una altitud de $149 \mathrm{msnm}$, con latitud de $29^{\circ} 00^{\prime} 53^{\prime}$ " y longitud de $111^{\circ} 07^{\prime} 56$," con una precipitación de $150 \mathrm{~mm}$ al año y temperatura promedio de $23^{\circ} \mathrm{C}$ y dentro del tipo de vegetación denominado matorral arbosufrutescente.

El área seleccionada es de dos hectáreas de terreno de agostadero del Campo Experimental, donde predomina un tipo de suelo denominado 
Migajon-Areno-Arcilloso y con una vegetación asociada de matorral con buffel (Cenchrus ciliaris L.), el que se acondicionó con labores de limpieza y cercada con alambres de púas (5 hilos), malla palomera de $50 \mathrm{~cm}$ de altura alrededor del área, para proteger a las plantas del daño de los roedores y lagomorfos (Figura 1).

Instalación del sistema de riego: Considerando las condiciones de baja humedad del área y con el fin de asegurar el establecimiento de las plantas, el futuro desarrollo y producción de semillas, se instaló un sistema de riego por goteo, para proporcionar riegos de auxilio a las plantas en los períodos más críticos del año, como también hacer más eficiente la aplicación y uso del agua.

Para la instalación del riego por goteo se empleó el siguiente material: manguera ciega p.e. de $16 \mathrm{~mm}$, gotero botón 4 lph, tubo pvc 3/4 c-40, cople T 3/4 X $15 \mathrm{~mm}$, tapón capa pvc 3/4" c- 40 , válvula pvc bla $3 / 4 \mathrm{~cm}$, filtro malla azud $3 / 4150 \mathrm{MESH}$, reducción campana pvc 1”x 33/4, tapón gotero y capuchón.

La realización de las zanjas, para la instalación de la manguera se utilizó un tractor equipado con vertedera, la zanja se hizo a una distancia aproximadamente de $50 \mathrm{~cm}$ de distancia del tronco (tallo) de las plantas. Estas mangueras están acondicionadas con tres goteros, separados cada uno a $40 \mathrm{~cm}$ para crear un bulbo de humedad en cada planta.

Aplicación de los riegos. Los riegos fueron aplicados 4 veces al mes con un lapso de tiempo de 4 horas cada uno, para generar un bulbo de humedad y mantener a las plantas en las mejores condiciones y asegurar su trasplante. A cada planta sele suministro aproximadamente 256 litros al año durante los meses de marzo, abril, mayo y junio.

Obtención de arbustos y árboles. Las especies utilizadas para el establecimiento del jardín botánico fueron propagadas y donadas por el vivero de la Comisión Nacional Forestal (CONAFOR Zona II) y vivero del Departamento de Agricultura. (Tabla 1)

Distribución de las especies en el área de estudio. Las especies que se utilizaron para este estudio fueron seleccionadas tomando en cuenta lo siguiente: la gustosidad por el ganado, el contenido de nutrientes y su estatus como especies clave en el estado de Sonora, con serios problemas de reducción en sus poblaciones naturales que las está llevando a un inminente peligro de extinción en sus áreas naturales.

La distribución de las especies de plantas en el área de estudio y de acuerdo al sorteo realizado las plantas arbustivas se encuentran al lado sur y los

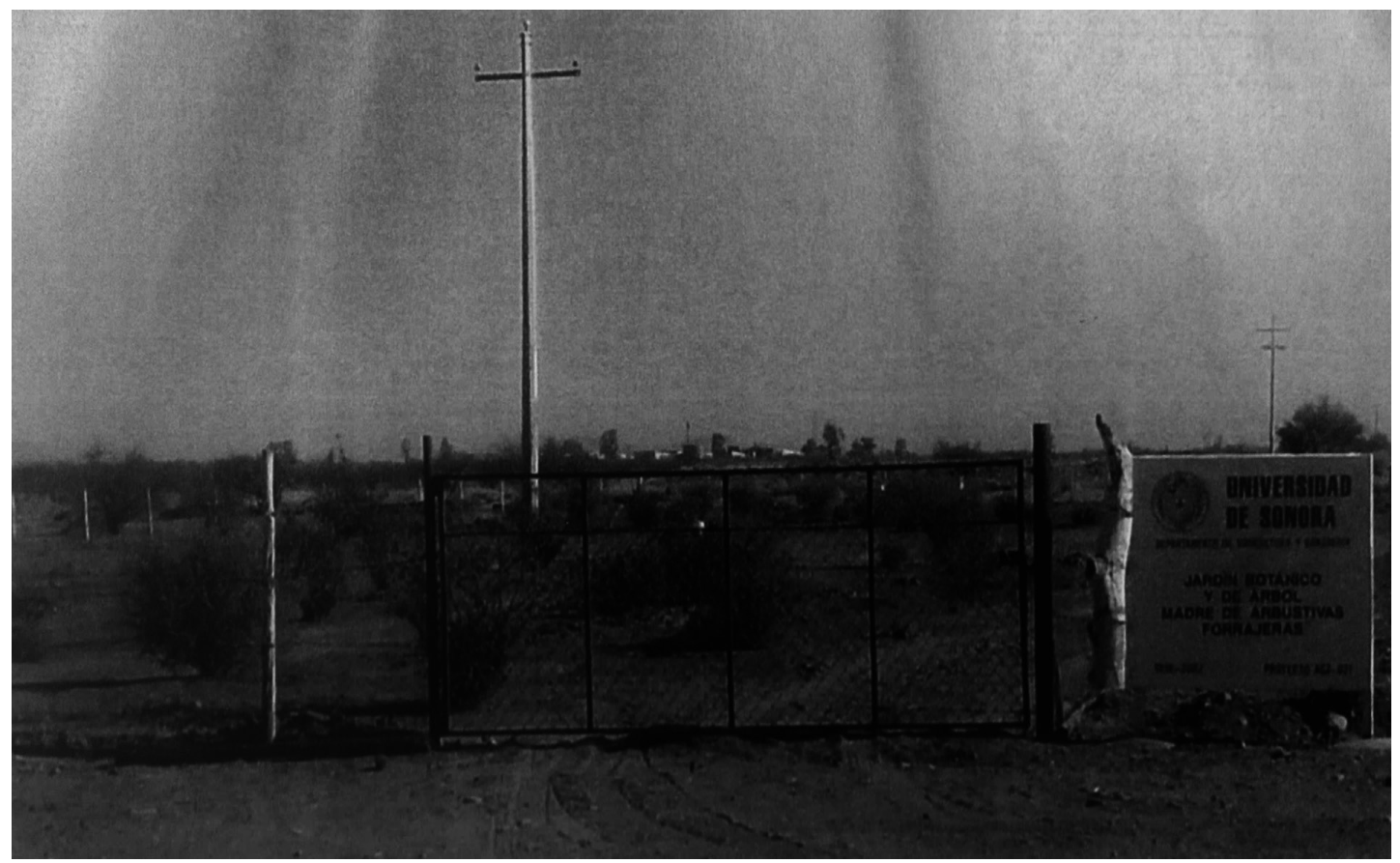

Figura 1. Jardín botánico y de árbol madre de arbustivas forrajeas. 
Tabla 1. Lista de las especies de arbustos y árboles en estudio.

\begin{tabular}{ll}
\hline Nombre común & \multicolumn{1}{c}{ Nombre científico } \\
\hline 1. Cosahui del norte & Calliandra eriophylla Benth \\
2. Cosahui del sur & Krameria parvifolia Benth \\
3. Chiltepin & Capsicum annuum Dunal \\
4. Chamizo cenizo & Atriplex canescens Pursh, Nutt \\
5. Sitiporo & Desmanthus couvillei (Britton \& Rose) Wiggins ex B.L. Turner \\
6. Jojoba & Simmondsia chinensis Link C. Karl. Schneid \\
7. Zámota & Coursetia glandulosa A. Gray \\
8. Piojito & Caesalpinia pumila (Britton \& Rose) F.J. Herm \\
9. Chamizo & Atriplex Polycarpa (Torr.) S. Watson \\
10. Orégano & Lippia palmeri S. Watson \\
11. Palo verde azul & Cercidium microphyllum (Torr) Rose y I.M. Johnst \\
12. Palo blanco & Ipomoea arborescens (Humb y Bonpl. Ex Willd) G. Don \\
13. Guayacan & Guaiacum coulteri A. Gray \\
14. Mezquite & Prosopis velutina Wooton \\
15. Palo fierro & Olneya tesota A. Gray \\
16. Palo verde chino & Cercidium floridum (Benth. ex A. Gray) S. Wats. \\
17. Guásima & Guazuma ulmifolia Lam \\
18. Mauto & Lysiloma divaricatum (Jacq.) J.F. Macbr \\
19. Pochote & Ceiba acuminata (S. Watson) Rose \\
20. Tepehuaje & Lysiloma watsonii Rose \\
\hline
\end{tabular}

árboles al lado norte. Las plantas presentaban una edad aproximada de 1 a 2 años antes de su trasplante.

\section{Trasplante de las especies}

Se utilizaron 10 ejemplares por especie tanto de arbustos como de árboles, para el trasplante se hicieron hoyos de aproximadamente un metro de profundidad; con una broca rotativa, posteriormente se le despojó la bolsa de plástico de la planta (dejando el cepellón) y se colocó en el centro del hoyo, se le coloco tierra, se aplastó la tierra, con el fin de retirar el aire que pudiera haberse almacenado y ocasionar que las raíces se sequen y por último se les aplicó un riego pesado para ayudar a la sobrevivencia y adaptación de las plantas, aplicándoseles un riego cada semana, durante 4 horas. La separación entre plantas fue de 3,5 metros entre cada planta tanto para árboles y arbustos.

\section{Parámetros a evaluar}

En el desarrollo de este estudio se realizaron dos tipos de actividades que permitieron alcanzar los objetivos planteados: Se instaló un sistema de riego que nos permitiera asegurar la sobrevivencia de las especies y producción de semillas y se plantaron 10 especies de arbustos y 10 de árboles.
Considerando el comportamiento especial de las especies en cuanto a su capacidad de adaptación y desarrollo en estos ambientes semidesérticos, se consideró adecuado evaluar cada una de las plantas en cuanto a sus características propias, midiéndose los siguientes parámetros: porcentaje de sobrevivencia, altura, diámetro de tallo, diámetro copa, fenología y el índice de crecimiento en \% de las especies en estudio.

Para el porcentaje de sobrevivencia se tomó en cuenta el número de plantas que se establecieron de cada una de las especies, después de los 15 días de su trasplante en \%. La medición dasométrica se llevó a cabo mediante la toma de datos de cada individuo midiendo el diámetro de tallo, diámetro de cobertura y la altura (Meza y Osuna, 2003). La medición del diámetro de tallo se realizó al ras del suelo, el diámetro del tallo y cobertura, se usó una cinta métrica expresada en cm y metros, para ello se utilizó la siguiente ecuación.

$$
\begin{aligned}
\mathrm{a}=\mathrm{Xd} * \pi / 2 \quad \text { Donde: } & \mathrm{a}=\text { Área } \\
& \mathrm{Xd}=\text { Diámetro } \\
& \pi=3,1416
\end{aligned}
$$

El índice de crecimiento se realizó de acuerdo a Carrillo et al., 2007, y se expresó en porcentaje y se calculó utilizando la ecuación: 


$$
\mathrm{IC}=\frac{\mathrm{Xf}-\mathrm{Xi} * 100}{\mathrm{Xf}}
$$

Donde:

IC $=$ Índice de crecimiento en $\%$.

$\mathrm{Xf}=$ Valor obtenido al final del período para altura, diámetro de copa o área basal.

$\mathrm{Xi}=$ Valor obtenido al inicio del período para altura, diámetro de copa o área basal.

Para las especies de arbustos y árboles se consideró el promedio de las especies que sobrevivieron al trasplante en el lapso de un año.

\section{Diseño experimental}

Para este trabajo se realizó un cuadro latino, tanto para las arbustivas como las arbóreas, con el propósito que no se repitiera la misma especie entre hileras y columnas para posteriormente realizar otros estudios, acerca de la preferencia del ganado por cada una de las especies ya establecidas, la influencia de la invasión del zacate buffel (Cenchrus ciliaris $\mathrm{L}$.) con respecto al desarrollo de las mismas, la influencia de la auto propagación por semillas de las especies en estudio con respecto a las otras especies, la capacidad de adaptación de los nuevos ejemplares, entre otros.

\section{Resultados}

\section{Sobrevivencia al trasplante de arbustivas}

La adaptación de las plantas arbustivas es muy importante sobre todo para los agostaderos de nuestra región. El comportamiento de adaptación y sobrevivencia al trasplante de las plantas arbustivas, fue satisfactorio mostrando de $90 \%$ de sobrevivencia para zámota, piojito y orégano, mientras que para sitiporo, cosahui del norte, y chamizo fue de un $80 \%$, y en el caso de chamizo cenizo, jojoba, chiltepín y cosahui del sur fue de $70 \%$, por lo tanto las arbustivas presentaron una excelente capacidad de adaptación y esto nos marca una respuesta favorable a la propagación mediante el trasplante cuando se maneja, con condiciones diferentes al manejo tradicional, si consideramos también que en este estudio la humedad con los auxilios de riego que fueron aplicados durante 4 meses.

Los resultados obtenidos en cuanto a la sobrevivencia al trasplante de las especies de chamizo fueron similares a los obtenidos por Loredo et al., (2007) donde ellos reportaron de un 60 a $90 \%$ de sobrevivencia al trasplante de chamizo (Atriplex canescens Pursh Nutt), al utilizar de 10 a 50 ton/ha de estiércol caprino. Parra y Sepulveda (1976), mencionan haber obtenido un $95 \%$ de sobrevivencia al evaluar el grado de adaptación como cultivo de la jojoba, dichos resultados son más atractivos que los obtenidos en este estudio y pudiera deberse a que los investigadores utilizaron plantas de jojoba más jóvenes por lo cual presentaron mejores resultados de adaptación que las plantas de más de un año de edad. Mc Caughey-Espinoza et al., (2016), evaluaron la sobrevivencia al trasplante del orégano como cultivo y obtuvieron un $95 \%$ de sobrevivencia al trasplante, con esto podemos señalar que los resultados son similares a los obtenidos en este estudio. Albella y Newton (2009), mencionan que existen valores arriba del $50 \%$ de sobrevivencia en algunas especies de arbustos de menos de 5 años de edad en el desierto de Mojave, y en lo que respecta a este estudio la mayoría de las especies de plantas de arbustos que fueron utilizadas presentaron en su primer año de establecimiento un excelente vigor en su adaptación por lo que podrían superar los 5 años de establecimiento en cuanto a la sobrevivencia que menciona Albella y Newton (2009), con la aplicación de riegos de auxilio en la época crítica del año.

\section{Altura de las plantas arbustivas}

$\mathrm{Al}$ realizar las mediciones de altura las plantas como el piojito, zámota y chamizo alcanzaron una altura arriba de 1,50 metros, mientras que las plantas de chamizo y jojoba presentaron alturas promedios de 1,49 y 1,12 metros y las plantas que presentaron menores alturas fueron, sitiporo, orégano, chiltepín, cosahui del sur y cosahui del norte, al no alcanzar a llegar a un metro de altura pero, a su vez, mostraron desarrollos normales para este tipo de arbustos que de acuerdo a sus condiciones en áreas silvestres donde su crecimiento es mucho menor (Tabla 2).

Turner et al. (2005) reportaron la altura de algunas especies arbustivas tales como, zámota (Coursetia glandulosa) que fue de 1,5 a 5 metros, piojito (Caesalpinia palmeri) 3 metros y Jojoba (Simmondsia chinensis) de 1 a 3 metros, tomando 
Tabla 2. Comportamiento en promedio de las plantas de las especies de arbustos.

\begin{tabular}{|c|c|c|c|c|c|c|}
\hline \multirow[b]{2}{*}{ Especies } & \multicolumn{3}{|c|}{ Trasplante } & \multicolumn{3}{|c|}{ Después del trasplante } \\
\hline & Altura metros & $\begin{array}{l}\text { Diámetro } \\
\text { de tallo } \mathrm{cm}^{2}\end{array}$ & $\begin{array}{c}\text { Diámetro } \\
\text { de copa } \mathrm{m}^{2}\end{array}$ & Altura metros & $\begin{array}{l}\text { Diámetro } \\
\text { de tallo } \mathrm{cm}^{2}\end{array}$ & $\begin{array}{l}\text { Diámetro } \\
\text { de copa } \mathrm{m}^{2}\end{array}$ \\
\hline Zámota & 1,22 & 16,13 & 1,24 & 1,61 & 20,76 & 2,11 \\
\hline Piojito & 1,23 & 9,01 & 1,17 & 1,62 & 13,07 & 1,94 \\
\hline Chamizo cenizo & 1,17 & 12,03 & 1,21 & 1,49 & 18,47 & 1,96 \\
\hline Jojoba & 0,89 & 6,11 & 0,99 & 1,12 & 10,75 & 1,39 \\
\hline Sitiporo & 0,64 & 6,91 & 0,31 & 0,83 & 14,52 & 0,95 \\
\hline Cosahui del norte & 0,39 & 4,90 & 0,32 & 0,47 & 7,87 & 0,63 \\
\hline Orégano & 0,43 & 6,17 & 0,53 & 0,77 & 10,54 & 1,27 \\
\hline Chiltepín & 0,37 & 4,23 & 0,41 & 0,72 & 6,28 & 0,64 \\
\hline Cosahui del sur & 0,36 & 4,81 & 0,35 & 0,45 & 7,63 & 0,68 \\
\hline Chamizo & 1,21 & 11,93 & 1,19 & 1,51 & 18,81 & 1,88 \\
\hline
\end{tabular}

en cuenta que dichas plantas ya son consideradas como plantas adultas. Por otra parte, Loredo et al. (2007), utilizaron estiércol de caprino obtuvieron una altura promedio de $57,3 \mathrm{~cm}$ en chamizo (Atriplex canescens Pursh Nutt). Mc Caughey-Espinoza et al. (2016), evaluaron la altura del orégano en dos sitios silvestres y cultivado, las plantas de los sitios silvestres presentaron alturas de 1,59 metro en Álamos y Puerto del orégano 0,92 metro, mientras que las plantas de orégano cultivado presentaron un promedio de 0,68 metro de altura durante los tres cortes que se les realizó, mientras en este estudio las plantas de orégano en promedio presentaron una altura de 0,77 metros.

\section{Diámetro de tallo de las arbustivas}

En lo que se refiere al incremento del diámetro de los tallos, en zámota, piojito, chamizo cenizo, sitiporo y chamizo presentaron un promedio, de $13,07,14,52,18,47,18,81$ y $20,76 \mathrm{~cm}^{2}$ en promedio respectivamente, de acuerdo al número de plantas sobrevivientes al trasplante, mientras que el resto de las especies de arbustivas como en el caso de jojoba, orégano, cosahui del norte, cosahui del sur y chiltepín presentaron de 10,75, 10,54, 7,87, 7,63 y $6,28 \mathrm{~cm}^{2}$ (Tabla 2). En las zonas áridas, el establecimiento y crecimiento de las plantas es muy variado de acuerdo con las especies de plantas e incluso entre las plantas de la misma especie, por lo tanto, una de las limitantes más importantes es el agua con la que no cuentan por períodos prolongados, en estos no hay presencia de lluvias.

\section{Diámetro de copa de las arbustivas}

Al evaluar el diámetro de copa algunas especies como, zámota, chamizo cenizo, piojito chamizo y jojoba, presentaron alturas de 2,11 a 1,39 $\mathrm{m}^{2}$, y quedando como rango intermedio el orégano con $1,27 \mathrm{~m}^{2}$ y el resto de las especies fue menor mostrando 0,64 a $0,95 \mathrm{~m}^{2}$ (Tabla 2). Mientras que Loredo et al., (2007), presentaron promedios de diámetro de copa en chamizo (Atriplex canescens Pursh Nutt) de 1,03 m², utilizando estiércol de caprino. Villa et al., (2011) en el cultivo de orégano (Lippia graveolens $\mathrm{HKB}$ ) y a los 140 días después del trasplante obtuvieron un diámetro de copa de $1,15 \mathrm{~m}^{2}$. Mc Caughey-Espinoza et al., (2016), al evaluaron el diámetro de copa del orégano de dos sitios silvestres y cultivado, obtuvieron que las plantas de los sitios silvestres presentaron un diámetro de copa en promedio de $1,12 \mathrm{~m}^{2}$ en Álamos y Puerto del orégano $0,92 \mathrm{~m}^{2}$, mientras que las plantas de orégano cultivado presentaron un promedio de $0,80 \mathrm{~m}^{2}$ de altura durante los tres cortes que se les realizo, mientras que en este estudio las plantas de orégano en promedio presentaron una altura de $1,27 \mathrm{~m}^{2}$. Si consideramos que las plantas en los dos sitios silvestres son plantas adultas ya establecidas y adaptadas a las condiciones adversas que existen en nuestra región y las de cultivo también a pesar que se les aplican riegos dichas plantas no presentan un diámetro de copa mayor a $1 \mathrm{~m}^{2}$, debido a que se podan (cosecha).

\section{Índice de crecimiento de las arbustivas}

Como una consecuencia directa con la aplicación de los riegos de auxilio para el 
establecimiento de las plantas arbustivas, se mostró un índice de crecimiento en el diámetro de tallo de 22,3 a 52,4\%, para el caso del diámetro de copa fue del 67,4 al 36,7\%, respectivamente, en el índice de crecimiento y por último la altura que fue de un 48,6 a un $19,9 \%$, dichos crecimientos en estos tres parámetros evaluados muestran claramente que al suministrar agua en épocas criticas del año las plantas reaccionan satisfactoriamente presentando un comportamiento de crecimiento mejor que el de las plantas que están en su hábitat natural, mostrando un mayor follaje y crecimiento, más kilogramos de materia fresca o seca, para el ganado doméstico o fauna silvestre, y tienen un alto valor nutricional considerándose una fuente de proteína de una excelente calidad y digestibilidad que caracteriza a las arbustivas forrajeras de nuestra región, como también más producción de semillas y de mejor calidad para futuras plantaciones en agostaderos deteriorados (Tabla 3 ).

\section{La sobrevivencia al trasplante de árboles}

Con respecto a la respuesta de las plantas de las diferentes especies de árboles que fueron trasplantados al final del estudio se obtuvo que estas presentaron una excelente adaptación que fue de 60 a $100 \%$ de sobrevivencias (Tabla 6), destacando especies como el palo blanco, mauto y tepehuaje los que obtuvieron $100 \%$ de sobrevivencia, seguidos de estos tenemos a palo verde chino, mezquite, palo fierro, palo verde azul con un $90 \%$ y guayacán con $80 \%$, y tenemos a la guasima y pochote que presentaron de $70 \mathrm{y}$ $60 \%$ de sobrevivencia. Esto nos indica que las especies de árboles que presentaron arriba del
$80 \%$ de sobrevivencia al trasplante presentan una gran capacidad para responder al manejo de programas de trasplantes en áreas protegidas. Por otra parte debemos de considerar que las especies que presentaron menor sobrevivencia al trasplante se puede decir que tienen diferentes necesidades para su adaptación.

Ríos et al. (2011) evaluaron la sobrevivencia en mezquite (Prosipis laevigata (H. \& B.) Jonhst), utilizando plantas con alturas de $45-60 \mathrm{~cm}$ y le aplicaron $20 \mathrm{~g}$ de hidrogel y obtuvieron una sobrevivencia al trasplante del $88,33 \%$, lo que podemos señalar que es similar a la que se obtuvo en este estudio como también de la importancia de mantener a las plantas hidratadas para obtener porcentajes arriba del $50 \%$ de sobrevivencia en el trasplante.

\section{Altura de las plantas de árboles}

También se evaluó el desarrollo para el grupo de especies de crecimiento arbolado (Tabla 4). en cuanto a la altura que alcanzaron durante el periodo de evaluación encontrándose un impacto mayor con el auxilio de riego, ya que, alcanzaron altura cercana a los 2 metros, como fue en el caso de palo verde chino, palo blanco, mezquite y palo verde azul con $1,97,1,95,1,93$ y 1,92 metros, respectivamente. Seguido de estos tenemos al pochote, guasima, mauto, y tepehuaje con alturas de $1,82,1,37,1,25$ y 1,17 metros. Las especies de árboles que presentaron más lento su desarrollo en cuanto altura fue el palo fierro con $0,90 \mathrm{~cm}$ y el guayacán con $0,47 \mathrm{~cm}$. A pesar que existieron plantas que no alcanzaron aproximadamente los 2 metros de altura podremos considerar que

Tabla 3. Índice de crecimiento en $\%$ de las especies de arbustos.

\begin{tabular}{lccc}
\hline \multirow{2}{*}{ Especies } & \multicolumn{3}{c}{ Índice de crecimiento en\% } \\
\cline { 2 - 4 } & Diámetro de tallo cm ${ }^{2}$ & Diámetro de Copa cm & Altura cm \\
\hline Zámota & 22,3 & 41,2 & 24,2 \\
Piojito & 31,1 & 39,7 & 24,1 \\
Chamizo cenizo & 34,9 & 38,3 & 21,5 \\
Jojoba & 43,2 & 28,8 & 20,5 \\
Sitiporo & 42,4 & 67,4 & 22,9 \\
Cosahui del norte & 37,7 & 49,2 & 17,0 \\
Orégano & 41,5 & 58,3 & 44,2 \\
Chiltepin & 32,6 & 35,9 & 48,6 \\
Cosahui del sur & 37,0 & 48,5 & 20,0 \\
Chamizo & 36,6 & 36,7 & 19,9 \\
\hline
\end{tabular}


Tabla 4. Comportamiento en promedio de las plantas de las especies de árboles.

\begin{tabular}{|c|c|c|c|c|c|c|}
\hline \multirow[b]{2}{*}{ Especies } & \multicolumn{3}{|c|}{ Trasplante } & \multicolumn{3}{|c|}{ Después del trasplante } \\
\hline & $\begin{array}{l}\text { Altura } \\
\text { metros }\end{array}$ & $\begin{array}{l}\text { Diámetro } \\
\text { de tallo } \mathrm{cm}^{2}\end{array}$ & $\begin{array}{l}\text { Diámetro } \\
\text { de copa } m^{2}\end{array}$ & $\begin{array}{l}\text { Altura } \\
\text { metros }\end{array}$ & $\begin{array}{l}\text { Diámetro } \\
\text { de tallo } \mathrm{cm}^{2}\end{array}$ & $\begin{array}{c}\text { Diámetro } \\
\text { de copa } \mathrm{m}^{2}\end{array}$ \\
\hline Palo verde chino & 1,33 & 22,16 & 1,68 & 1,97 & 28,34 & 2,58 \\
\hline Palo blanco & 1,41 & 19,13 & 2,07 & 1,95 & 31,93 & 2,78 \\
\hline Guayacán & 0,38 & 0,67 & 0,54 & 0,47 & 0,85 & 0,62 \\
\hline Mezquite & 1,31 & 23,42 & 1,89 & 1,93 & 30,68 & 2,82 \\
\hline Palo fierro & 0,69 & 6,12 & 0,81 & 0,90 & 7,45 & 0,98 \\
\hline Palo verde azul & 1,41 & 22,94 & 1,36 & 1,92 & 29,28 & 2,65 \\
\hline Guasima & 0,98 & 4,13 & 1,53 & 1,37 & 17,16 & 2,25 \\
\hline Mauto & 0,89 & 0,97 & 0,42 & 1,25 & 4,21 & 1,09 \\
\hline Tepehuaje & 0,64 & 0,89 & 0,64 & 1,17 & 1,11 & 1,06 \\
\hline Pochote & 1,48 & 19,52 & 1,56 & 1,82 & 23,15 & 2,01 \\
\hline
\end{tabular}

dichas alturas son superior al desarrollo normal de dichas especies en los agostaderos, por lo tanto, el proporcionarles agua en la época crítica del año fue muy satisfactorio al ver resultados.

De acuerdo con los resultados obtenidos por García et al. (2014), en mezquite (Prosopis glandulosa Torr.) de una edad de 11 años (5 años en vivero y 6 de establecimiento) presentaron una altura promedio de 3,719 metros. Dichos resultados difieren con los obtenidos en este estudio ya que las plantas de mezquite (Prosopis velutina Wooton) que se utilizaron tenían una edad de 3 años aproximadamente ( 2 años en vivero y 1 año de establecimiento), y presento una altura promedio de 1,93 metros. Mientras que Ríos et al. (2011), presentan alturas en mezquite (Prosipis laevigata (H. \& B.) Jonhst) de 52,3-87,8 cm, dichos datos nos muestran que estas plantas son plantas jóvenes y la medición fue a los 3 meses después del trasplante, mientras que las plantas de mezquite (Prosopis velutina Wooton) que se utilizaron en este estudio presentaron una altura promedio de 1,93 metros al año de su trasplante por lo que presentaron un excelente comportamiento con el auxilio del riego.

Turner et al. (2005) evaluaron la altura en árboles de Ipomea arborescens y encontraron plantas de más de 12 metros, en palo fierro (Olneya tesota) de 5-10 metros, palo verde azul (Cercidium microphyllum) 6 metros y palo verde chino (Cercidium floridum) más de 12 metros. Si consideramos algunas de las especies de árboles evaluados en este estudio se muestra claramente que palo blanco, palo fierro, palo verde azul y palo verde chino presentaron excelentes alturas a pesar de la edad, en el caso de palo fierro es una especie de lento crecimiento.

\section{Diámetro de tallo de los árboles}

En lo que se refiere al incremento del diámetro del tallo de las especies arbóreas el palo blanco, presentó mayor diámetro en promedio de $31,93 \mathrm{~cm}^{2}$, seguido de este se encuentra al mezquite con $30,68 \mathrm{~cm}^{2}$, y palo verde azul con $29,28 \mathrm{~cm}^{2}$, y palo verde chino con $28,34 \mathrm{~cm}^{2}$, quedando en un rango intermedio, el pochote con un promedio de $23,15 \mathrm{~cm}^{2}$ y la guasima con $17,16 \mathrm{~cm}^{2}$, mientras que el guayacán, tepehuaje, mauto y palo fierro, registraron menor incremento en el diámetro tallo con promedios de $0,85,1,11,4,21,7,45 \mathrm{~cm}^{2}$, lo que puede atribuirse a que las especies de guayacán y palo fierro, son árboles que presentan diferentes comportamientos entre sí y estos dos últimos tienen un lento crecimiento, comparado al resto de las especies de árboles en estudio y en el caso del mauto y tepehuaje son plantas jóvenes de un año de edad aproximadamente (Tabla 4).

Al comparar los resultados con los de Ríos et al. (2011), evaluaron el diámetro de tallo en mezquite (Prosipis laevigata (H. \& B.) Jonhst), obteniendo $8,63 \mathrm{~cm}^{2}$ a los 63 días después del trasplante utilizando $20 \mathrm{~g}$ de hidrogel por planta. García et al. (2014), reportaron un diámetro de tallo en mezquite (Prosopis glandulosa Torr.), de $93,415 \mathrm{~cm}^{2}$, dichos árboles se podrían considerar como adultos y tomando en cuenta que el sitio de plantación fue un área agrícola, con una precipitación pluvial anual de $648 \mathrm{~mm}$, por lo que los datos no son similares a los obtenidos en este estudio tomando en cuenta que en nuestra región tenemos una precipitación pluvial de $150 \mathrm{~mm}$ al año por lo que obtuvimos un diámetro de tallo en mezquite (Prosopis velutina Wooton) de $30,68 \mathrm{~cm}^{2}$. 
Cabe señalar que los resultados que obtuvimos en este estudio, los árboles mostraron un excelente tamaño (vigor) obteniéndose buenos diámetros de tallos, por tanto, es importante señalar que la aplicación de los riegos, se reflejó notoriamente en la adaptación de las especies en el trasplante como, también tener el área protegida para evitar la presencia de ganado y lagomorfos.

Para obtener resultados a corto o mediano plazo de las áreas degradadas es importante enfocarnos en trabajos que nos permitan obtener resultados más precisos, y para ello es importante utilizar especies de la región ya, que son las que se adaptan con mayor facilidad a las condiciones existentes (altas y bajas temperaturas, escasas precipitaciones pluviales, plagas de insectos y enfermedades) lo que las hace menos vulnerables a morir como, también ir concientizando a las personas de la importancia de dichas especies maderables y no maderables en nuestros ecosistemas, para asimismo ir cambiando la cultura ambiental, la que ha sido uno de los principales problemas con los que nos enfrentamos día a día, y también mantener nuestros ecosistemas naturales.

\section{Diámetro de copa de las plantas de árboles}

Con respecto al desarrollo foliar de estas especies de árboles se puede observar (Tabla 4), donde plantas de palo blanco, palo verde azul, palo fierro y palo verde chino, mostraron una excelente cubierta vegetal con 2,78, 3,65, 3,12 y $2,58 \mathrm{~m}^{2}$, respectivamente que demostraron un excelente desarrollo de estas especies cuando tienen humedad disponible a través del año. Asimismo sus tallos alcanzaron un buen desarrollo mostrando una base definida y vigorosa donde destacan estas mismas especies.

García et al. (2014), evaluaron en diámetro de copa en árboles de mezquite (Prosopis grandulosa Torr.) obteniendo un promedio de $17,88 \mathrm{~m}^{2}$, al compararlos con los obtenidos de este estudio el mezquite (Prosopis velutina Wooton) mostró un diámetro de copa de 2,82 $\mathrm{m}^{2}$, dichos resultados difieren mostrando una gran diferencia y puede deberse a la edad de los árboles, García et al., 2014, utilizaron árboles de una edad de 11 años mientras que los árboles en estudio llegaron a una edad de 3 años al ser evaluados, ente otros factores.

A pesar de que son plantas del desierto sonorense, que requieren poca agua son consideradas, por los resultados que se obtuvieron en este estudio, podemos mencionar que es importante la disponibilidad de agua para el establecimiento y crecimiento de las especies, ya que estas en su estado silvestre su crecimiento es lento aunado al pastoreo de animales silvestres o domésticos, baja precipitación, podas inmoderadas e incendios forestales lo que propicia un estrés en las plantas y puede llegar en un momento dado a que estas mueran.

\section{Índice de crecimiento de los árboles}

También se presentó en el caso de las especies de árboles en este trabajo un importante índice de crecimiento (Tabla 5) en cuanto al diámetro de tallo, mauto presentó un $77,0 \%$, guasima $75,9 \%$, palo blanco $40,1 \%$, y los que presentaron un índice de crecimiento medio fue para el resto de las especies con 15,2 a $23,7 \%$. En lo que comprende para el índice de crecimiento del diámetro de copa fue para

Tabla 5. Índice del crecimiento en \% de las especies de árboles.

\begin{tabular}{lccc}
\hline \multirow{2}{*}{ Especies } & \multicolumn{3}{c}{ Índice de crecimiento en\% } \\
\cline { 2 - 4 } & Diámetro de tallo $\mathrm{cm}^{2}$ & Diámetro de copa $\mathrm{m}^{2}$ & Altura cm \\
\hline Palo verde chino & 21,8 & 34,9 & 82,5 \\
Palo blanco & 40,1 & 35,5 & 82,3 \\
Guayacán & 21,2 & 12,9 & 19,1 \\
Mezquite & 23,7 & 33,0 & 32,1 \\
Palo fierro & 17,9 & 17,3 & 23,3 \\
Palo verde azul & 21,7 & 48,7 & 26,6 \\
Guasima & 75,9 & 32,0 & 28,5 \\
Mauto & 77,0 & 61,5 & 21,6 \\
Tepehuaje & 19,8 & 39,6 & 45,3 \\
Pochote & 15,2 & 22,4 & 7,7 \\
\hline
\end{tabular}


mauto $61,5 \%$, palo verde azul $48,7 \%$ y tepehuaje $39,6 \%$, seguidas de estas tenemos árboles que presentaron de $12,9 \%$ a $32,0 \%$ y en altura tenemos al palo verde chino con un $82,5 \%$ y palo blanco con $82,3 \%$, siendo estos los que presentaron un mejor índice de crecimiento en cuanto a altura, el resto de las especies de árboles mostraron de 7,7 a $45,3 \%$. De acuerdo con los resultados obtenidos en cuanto al porcentaje del índice del crecimiento de los árboles podemos señalar que fueron favorables al presentar en los tres parámetros evaluados un claro crecimiento mostrando un tallo más firme, mejor altura y más follaje lo que es ideal para establecer programas de reforestación en áreas perturbadas del estado de Sonora, que nos permitan a corto o mediano plazo tener áreas rehabilitadas con especies nativas (endémicas) y que presentan un contenido nutricional muy importante en cuanto a la producción de forraje y, por ende, se produciría mayor cantidad de carne o leche, como también, tener mejor porcentaje de apariciones, logrando que la actividad ganadera del estado sea más rentable. Como también sin dejar a un lado, los beneficios que estas especies endémicas nos proporcionan gratuitamente a nuestro ecosistema.

De acuerdo con todo lo anterior, es de gran importancia tomar interés en trabajar con plantas maderables y no maderables, las que nos genera una gran variedad de productos importantes para la existencia de los seres vivos. Las especies de plantas utilizadas en este estudio, para algunas de ellas fue imposible plantearlas en los resultados por tener nula información y de otras especies existe una escasa información sobre estudios similares con dichas especies.

\section{Época de floración de arbustivas y árboles en estudio}

La mayoría de las especies presentaron una madurez adecuada para iniciar su etapa de floración como, fue el caso de las especies de zámota, orégano, chiltepín, palo blanco entre otros presentaron más de 3 veces al año floración y se atribuye a los riegos que se le aplicaron en las épocas críticas del año, lo que es muy importante ya que esto permitirá tener suficiente material vegetal para realizar futuras propagaciones con estas especies $\mathrm{y}$ asegurando el material genético. Cabe mencionar que este tipo de plantas silvestres, algunas de ellas, presentan floración de una a dos veces al año, como también la producción de semilla varía año con año, y esto depende de la época de lluvias. En el mes de noviembre las plantas de palo blanco, palo verde chino, mezquite, palo fierro, palo verde azul, pochote, mauto, cosahui del sur, cosahui del norte, zámota, piojito, orégano, chamizo, chiltepín y sitiporo presentaron floración. En el mes de febrero: jojoba, chamizo cenizo, zámota, piojito, orégano, chiltepín. En marzo: palo blanco, palo verde chino, mezquite, palo fierro, tepehuaje, jojoba, orégano, chamizo y sitiporo. Abril: cosahui del norte, jojoba, chamizo cenizo, zámota, piojito, orégano, chamizo y chiltepín. Junio tepehuaje, orégano y chiltepín y en el mes de julio fueron: palo blanco, palo verde chino, mezquite, palo fierro, piojito, orégano, chamizo y chiltepín, cosahui del sur, guayacán y mauto, dichas especies presentaron floración.

Los resultados obtenidos en este estudio son muy importantes debido a que los cambios fisiológicos de cada una de las especies de nuestra región han sido poco estudiadas lo que nos indica no se les ha dado la importancia que estas tienen en nuestros ecosistemas naturales, por lo que es complicado realizar comparaciones.

\section{Conclusiones y Recomendaciones}

El sistema de riego presurizado estimuló a las plantas de árboles y arbustos permitiendo asegurar su trasplante y su floración en más épocas del año y también presentaron buen desarrollo tanto de altura, diámetro de copa y tallo, por lo anteriormente mencionado esto nos genera más producción de semilla.

Se recomienda que al plantar la especie ya sea árbol o arbusto tengan un promedio de aproximadamente dos años de edad, ya que con esto se obtiene un $90 \%$ de sobrevivencia en el trasplante independientemente de la procedencia de las especies.

Cercar bien el área experimental con malla ciclónica, ya que esta ayudará a proteger mejor a las especies en estudio de los roedores.

Dar a conocer la importancia que estas especies representan como plantas forrajeras, como restauradoras del medio ambiente, por la fisonomía y restauración del suelo y como medicinales.

Utilizar las semillas que se produzcan en el jardín botánico de las especies establecidas, en las instituciones gubernamentales o por la misma institución para seguir estudiando estas especies, 
Esto con el fin de investigar la viabilidad de la semilla, o de reforestar áreas deterioradas y establecer una área con plantas de gran importancia forrajera para nuestra región es una opción que se tiene para evitar la desaparición de dichas especies y así asegurar el germoplasma para establecer plantaciones en los agostaderos los que están explotados o abandonados, también para conocer y entender más sobre nuestra flora endémica, de la cual nos falta mucho por aprender.

\section{Literatura Citada}

Abella, S.R. \& Newton, A.C.

2009. A systematic review of species performance and treatment effectiveness for revegetation in the Mojave Desert, USA. In: Fernandez-Bernal, A. and De La Rosa, M.A. (eds). Arid environments and wind erosion. Nova Science Publishers, Inc. Hauppauge, NY, US. pp. 45-74.

Carrillo Flores, R.; Gómez Lorence, F.; Arreola Ávila, J.G.

2007. Efecto de poda sobre potencial productivo de mezquitales nativos en la comarca lagunera, México. Unidad Regional Universitaria de Zonas Áridas. Bermejillo, Dgo. Revista Chapingo Serie Zonas Áridas, 6: 47-54 p.

García, M.G.E.; Jiménez, P.J.; Aguirre, C.O.A.; González, R.H.;

Carrillo, P.A.; Espinosa, R.M. y García, G.D.A.

2014. Biomasa de dos especies de matorral en tres densidades de plantación en Tamaulipas, México. Revista Latinoamericana de Recursos Naturales, 10 (2): 52-59 p.

Ibarra, F.; Martín, M.H.; Moreno, M.S.; Denogean, F.G. y

Gerlach, L.

2005. El zacate buffel como una alternativa para incrementar la rentabilidad de los ranchos en la zona serrana de Sonora. Revista Mexicana de Agronegocios, 9 (16): 521-529.

Jaramillo, V.V.

1994. Revegetación y reforestación de las áreas ganaderas en las zonas templadas de México. Ed. INCA RURALCOTECOCA. México, 40 p.

Loredo O. C.

1996. Evaluación de técnicas culturales en Atriplex canescens. XII Congreso Nacional sobre Manejo de Pastizales. Zacatecas, Zac. México. 53 p.
Parra, H. y Sepúlveda, B.

1976. Avances de la investigación sobre la jojoba en el estado de Baja California Sur. Memorias de la II Conferencia Internacional sobre la Jojoba y su Aprovechamiento, 10-12 de febrero de 1997. CONACYT. Ensenada B.C.N México. 25-36 p.

Mc Caughey-Espinoza, D. M.; Ortega-Nieblas, M.M. y RoblesBurgueño, M.R.

2016. Establecimiento del cultivo del Orégano Lippia palmeri Watson calidad del aceite esencial. X Simposio Internacional sobre Flora Silvestre en Zonas Áridas. 12-14 de octubre 2016, 163-173 p.

Meza, S.R.; Osuna, E.L.

2003. Estudio dasométrico del mezquite en la zona de las pocitas, B.C.S. SAGARPA-INIFAP-CIRNOR. México. Folleto científico, 3: 1-7 p.

Ríos, S.J.; Rivera, G.M.; Valenzuela, N.L.; Trucios, C.R. y Rosales, S.R.

2011. Diagnosis of mesquite reforestation and methods for increasing survival in Durango, Mexico. Revista Chapingo serie Zonas áridas, 11 (2): 53-67 p.

Turner, R.M.; Bowers, J.E. \& Burgess, T.L.

2005. Sonoran Desert plants: an ecological atlas. University of Arizona Press. Tucson, AZ. 501 p.

Villa-Castorena, M.; Catalán-Valencia, E.A.; Arreola-Ávila, J.G.; Inzunza-Ibarra, M.A.; López, A.R.

2011. Influencia de la frecuencia del riego en el crecimiento de orégano (Lippia graveolens $\mathrm{HKB}$ ). Revista Chapingo, serie ciencias forestales y del ambiente, 17 (spe): 183-193. 
